\title{
Identification and expression analysis of nitric oxide synthase gene, Mj NOS, in kuruma shrimp Marsupenaeus japonicus
}

\author{
M. Inada ${ }^{1}$, T. Mekata ${ }^{2}$, R. Sudhakaran ${ }^{3}$, S. Okugawa ${ }^{1}$, A.M. El Asely ${ }^{1}$, \\ Nguyen T. H. Linh', T. Yoshida ${ }^{3}$, T. Kono ${ }^{3}$, M. Sakai ${ }^{3}$, T. Yui ${ }^{4}$ and T. \\ $\operatorname{Itami}^{3} *$
}

${ }^{1}$ Graduate School of Agriculture, University of Miyazaki, 1-1, Gakuen Kibanadai-nishi, 8892192 Miyazaki, Japan

${ }^{2}$ Interdisciplinary Graduate School of Agriculture and Engineering, University of Miyazaki, 1-1, Gakuen Kibanadai-nishi, 889-2192 Miyazaki, Japan

${ }^{3}$ Faculty of Agriculture, University of Miyazaki, 1-1 Gakuen Kibanadai-nishi, Miyazaki, 8892192 Miyazaki, Japan

${ }^{4}$ Faculty of Engineering, University of Miyazaki, 1-1 Gakuen Kibanadai-nishi, Miyazaki, 8892192 Miyazaki, Japan

\begin{abstract}
Nitric oxide (NO) signaling is involved in many physiological processes in vertebrates and invertebrates. In mammals, NO plays as an endothelium-derived relaxing factor in the form of a simple unstable gas, as a neurotransmitter in the central and peripheral nervous systems and as an immune effector mediated by macrophages. In crustaceans, nitric oxide synthase (NOS) plays a significant role in innate immunity and in the regulation of the nervous system. We describe the full-length cDNA sequence $(4,616 \mathrm{bp})$ of the kuruma shrimp M. japonicus $\mathrm{NOS}(\mathrm{Mj}$ NOS). The open reading frame of Mj NOS encoded a protein of 1,187 amino acids with an estimated mass of $134 \mathrm{kDa}$, and had an $82.3 \%$ sequence homology with the NOS gene of the land crab Gecarcinus lateralis. In the brain, gill, intestine, thoracic ganglion and testis of the kuruma shrimp Mj NOS, mRNA was constitutively expressed. When Vibrio penaeicida was injected into the kuruma shrimp, Mj NOS was expressed in the brain, gill, heart, lymphoid organ, intestine and thoracic ganglion. In the gill, Mj NOS expression reached its peak at $12 \mathrm{~h}$ and decreased to its normal level $24 \mathrm{~h}$ after $V$. penaeicida injection.
\end{abstract}

\section{Introduction}

In vertebrates, nitric oxide (NO) serves as a primary immune activator molecule and a signal messenger with multifaceted roles (Aktan 2004; Bogdan et al. 2000). In invertebrates, NO is predominantly associated with antibacterial, antiviral and antiparasitic interactions as a cytotoxic molecule functioning directly or after 
interaction with other free-radical intermediates (Torreilles and Guérin 1999; Nappi 2000 et al.; Beck et al. 2001; Jiang et al. 2006). NO works as an endothelium-derived relaxing factor in the form of a simple unstable gas (Palmer et al. 1987; Furchgott 1990) as a neurotransmitter in the central and peripheral nervous systems (O'Dell et al. 1991) and as an immune effector mediated by macrophages in mammals (Nathan and Hibbs 1991; Fang 1997).

NOS has a C-terminal reductase domain and an $\mathrm{N}$ - terminal oxygenase domain. The C-terminal reductase domain has a binding motif for flavin mononucleotide (FMN), flavin adenine dinucleotide (FAD) and nicotinamide adenine dinucleotide phosphate (NADPH). The N-terminal oxygenase domain has a binding motif for $\mathrm{P} 450$ like cysteine thiolate-ligate heme and tetrahydrobiopterin $\left(\mathrm{BH}_{4}\right)$. Both the oxygenase and reductase domains are linked by a calmodulin (CaM) binding motif (Nathan 1992; Schmidt et al. 1992; Andrew and Mayer 1999; Nishida et al.1992).

NOS has three isoforms in mammals. They are neuronal NOS (nNOS), endothelial NOS (eNOS) and inducible NOS (iNOS). By the concentration of intracellular $\mathrm{Ca}^{2+}$ and binding with CaM, nNOS and eNOS are constitutively expressed and regulated. In contrast, iNOS lacks an autoinhibitory loop and binds with CaM at a high affinity and low $\mathrm{Ca}^{2+}$ levels; this action is predominantly regulated at the transcriptional level (Andrew and Mayer 1999). Once binded with the NOS enzyme, $\mathrm{CaM}$ stimulates the rate of electron transfer from the reductase domain to the oxygenase domain (Abu-Soud and Stuehr 1993; Abu-Soud et al. 1994).

Insect and vertebrate NOS genes have a high sequence homology with the invertebrate NOS gene and have proved to be involved in many physiological reactions (Luckhart et al. 1998). In the fruit fly Drosophila melanogaster and the tobacco hornworm Manduca sexta, NO regulates the morphogenesis of the nervous system (Gibbs and Truman 1998; Truman et al. 1996). As a neurotransmitter, NO also functions in the central nervous system and in the antennal lobe of the honeybee Apis mellifera and fruit fly (Müller 1996; Nighorn et al. 1998).

In crustaceans, signal transduction using NO and cyclic guanosine monophosphate (cGMP) plays a role in neuronal development and in neuron and cardiac muscle regulation. In crabs, the NO/cGMP signaling pathway is essential for the systematic assembly of the neuronal circuit that drives rhythmic movements (Scholz et al. 2001; Scholz et al. 2002). NOS is found in the land crab Gecarcinus lateralis, a crustacean species with a high NOS gene sequence homology with the insects. In addition, Gecarcinus lateralis NOS shows significantly high NOS mRNA expression levels in the ovary, testis and eyestalk ganglia (Kim et al. 2004). 
In Fenneropenaeus chinensis and the kuruma shrimp $M$. japonicus, NOS activity during viral infection has been studied (Jiang et al. 2006). However, the fulllength cDNA of NOS from shrimp has yet to be reported. Additionally, only the Litopenaeus vannamei EST database encoding the partial NOS gene had been registered at the time of this study. Consequentially, the cloning of the full-length cDNA of Mj NOS comprises the first cloning report on the shrimp NOS gene. In this report, we describe the full-length cDNA encoding of the NOS gene from the kuruma shrimp denoted as Mj NOS. Under the auspices of our research, we investigated the gene expression after $V$. penaeicida injection.

The shrimp culture industry has experienced worldwide growth since the 1970's (Momoyama and Muroga 2005). With its expansion, various viral and bacterial diseases that have caused serious damage to the shrimp culture industry have been reported (Momoyama and Muroga 2005). V. penaeicida infection was spread from the late 1980's to the early 1990s' and caused an average 20-30\% loss of shrimp production annually (Momoyama and Muroga 2005). This indicates that $V$. penaeicida infection is a serious problem in the shrimp culture industry.

\section{Materials and Methods}

\section{Animals}

Adult kuruma shrimp, M. japonicus (average weight: $15 \mathrm{~g}$ ) were obtained from a shrimp farm in Miyazaki, Japan. They were acclimatized in aerated seawater at $22{ }^{\circ} \mathrm{C}$ and fed a commercial diet equaling $1 \%$ of their body weight once daily.

\section{Designing of degenerate primers}

A partial gene of NOS cDNA was initially obtained by RT-PCR with degenerate primers. It was designed from the conserved regions of the land crab $(G$. lateralis) NOS gene and L. vannamei in the EST database (GenBank accession numbers: AY552549 and FE061797) using ClustalW alignment with the ClustalW program (Hall 1999). The degenerate primers NOS-F1 and NOS-R1 (Table 1) were designed to anneal DNA sequences encoding the LALSREP and QMRDEN region, respectively. Additionally, another set of degenerate primers, NOS-F2 and NOS-F3 (Table 1), was designed on the basis of the conserved regions. It aligned with the lobster, parasitic bee and honeybee NOS genes in the EST databases (GenBank accession numbers: EW703101, GE409919, and NM_00101296, respectively) to anneal the DNA sequences encoding TELVYGAK and WSKLQVFD, respectively. 


\section{RNA extraction and cDNA preparation}

Total RNA was extracted from a pool of gill tissues of three individual kuruma shrimp using Trizol Reagent (Invitrogen, USA) in accordance with the manufacturer's instructions. It was quantified using a NanoDrop spectrophotometer (Thermo Fisher Scientific, USA). Purity was determined by measuring $\mathrm{OD}_{260 \mathrm{~nm}} / \mathrm{OD}_{280 \mathrm{~nm}}$. The RNA samples were treated with RNase free DNase I (Invitrogen, USA) and cDNA was synthesized using a ReverTra Ace qPCR RT Kit (Toyobo, Japan) in accordance with the manufacturer's instructions. It was used as the template for PCR analysis.

\section{Cloning and sequencing}

Initially, on the conserved regions of the crustacean NOS domain, one set of degenerate primers, NOS-F1 and NOS-R1 (Table 1) was designed. PCR was performed with cDNA prepared using these primers to amplify the initial predicted sequence. Two forward degenerate primers, NOS-F2 and NOS-F3 (Table 1) were designed from three invertebrate NOS genes to perform RACE-PCR using a SMART RACE cDNA Amplification Kit (Takara Bio, Inc., Japan) in accordance with the manufacturer's instructions (Takara Bio, Inc., Japan). This was used to further identify the gene sequences. Following the determination of a partial sequence of the NOS gene, the entire length was obtained using 5'- and 3'-RACE-PCRs with the gene-specific primers (Table 1). The PCR products were cloned into the pGEM-T Easy vector (Promega, USA). They were then transformed into DH5 $\alpha$ (Promega, USA). Recombinants were identified using the red-white color of colonies on MacConkey agar (Sigma-Aldrich, USA). Plasmid DNA from at least three independent clones was recovered using the QIAprep Spin Miniprep Kit (QIAGEN, Japan). Sequencing was performed using a CEQ 8000 Automated Sequencer (Beckman Coulter, Inc., USA).

The structural domains and signal peptide of Mj NOS amino acid sequences were predicted using a simple modular architecture research tool (SMART; Version 6.0) (http://smart.embl-heidelberg.de/help/smart_about.shtml). The sequence generated was analyzed for similarity with other known sequences. FASTA and the basic alignment search tool suite of the MatGat 2.02 program were used. Direct comparisons between cDNA sequences were performed using the gap program of BioEdit where multiple sequence alignments were generated using Clustal W (http://www.ebi.ac.uk/clustalw/). A phylogenetic chart was constructed. Full-length amino acid sequences of previously published NOS molecules using the neighborjoining (NJ) method with MEGA 4 (http://www.megasoftware.net) were used for phylogenetic analysis. 
Table 1. PCR primers used for Mj NOS analysis.

\begin{tabular}{|c|c|}
\hline Primers & Sequence $\left(5^{\prime}-3^{\prime}\right)$ \\
\hline \multicolumn{2}{|c|}{ Degenerate PCR } \\
\hline NOS-F1 & TGGCCCTGTCTCGTGAAC \\
\hline NOS-R1 & GTTTTCATCCCKCATCTG \\
\hline NOS-F2 & AGAAYTGGTTTWCGGMGCTA \\
\hline NOS-F3 & GGTCHAAGTTACAGGTVTTCGA \\
\hline \multicolumn{2}{|l|}{ 5'-RACE } \\
\hline $5^{\prime}-\mathrm{R} 1$ & CTGACATTGCTGAGAGGACCAAAGT \\
\hline $5^{\prime}-\mathrm{R} 2$ & ACTGGATACGGCCGATACACCTT \\
\hline \multicolumn{2}{|l|}{ 3'-RACE } \\
\hline 3'-F1 & CCAGGATCTTCTTGTTGGTGTTGG \\
\hline $3^{\prime}-\mathrm{F} 2$ & GTGTACCGTCAAGTTGTAGAGCAGAAGG \\
\hline $3^{\prime}-\mathrm{F} 3$ & AAAGTGCAAGAGTGAAGATGTCC \\
\hline \multicolumn{2}{|c|}{ Cloning for partial sequence primer } \\
\hline $\mathrm{c}-\mathrm{R} 1$ & CGACCATGCTCCTGGACAATAGACT \\
\hline $\mathrm{c}-\mathrm{R} 2$ & CACACATTCAGCCATGGTACAGTCAC \\
\hline c-R3 & CACCATAATGTAGTGGTCCCTTTCCA \\
\hline $\mathrm{c}-\mathrm{R} 4$ & GTCAGCTTCACTAGCCTCTC \\
\hline $\mathrm{c}-\mathrm{F} 1$ & CTTCAACGGCTGGTACATGGTGT \\
\hline $\mathrm{c}-\mathrm{F} 2$ & GTTGGTGGTCACGTCTACTT \\
\hline \multicolumn{2}{|c|}{ RT-PCR analysis } \\
\hline Mj NOS-F & GCCCTGTCTCGTGAACCTAC \\
\hline Mj NOS-R & TTTTCATCCCTCATCTGTAGCA \\
\hline $\mathrm{Mj} \mathrm{EF} 1 \alpha-\mathrm{F}$ & GTCTTCCCCTTCAGGACGTA \\
\hline Mj EF1 $\alpha-R$ & GAACTTGCAGGCAATGTGAG \\
\hline
\end{tabular}

\section{Mj NOS expression by RT-PCR}

Various tissues from body parts including the brain, gill, heart, hemocytes, hepatopancreas, intestine, lymphoid organ, muscle, thoracic ganglion, stomach, hematopoietic organ, ovary and testis were obtained from healthy shrimp $(n=3)$. Vibrio penaeicida was obtained from the National Institute of Technology and Evaluation (NITE) Biological Resource Center (NBRC No. 15640). It was cultured in marine broth (Becton, Dickinson, USA) liquid medium. The concentration of the bacterial solution was adjusted at $2 \times 10^{5} \mathrm{CFU}^{-1}$ on the basis of the McFarland No. 1. For the expression analysis of the Mj NOS gene, $100 \mu \mathrm{L}$ of the bacterial solution was injected into second abdominal segment of each shrimp $(n=6)$ and PBS was injected for control. Total RNA was extracted from each organ using Trizol Reagent in accordance with the manufacturer's instructions. The cDNA was synthesized using a ReverTra Ace qPCR 
RT Kit after DNase I treatment. The gene-specific primers Mj NOS-F and Mj NOS-R (Table 1) were designed and used to amplify the conserved regions in the reductase domain of the Mj NOS gene. The kuruma shrimp elongation factor $1 \alpha(\mathrm{EF} 1 \alpha)$ gene (Table 1: Mj EF1 $\alpha-F$, -R) served as the control to confirm the quality and quantity of the cDNA used. A time course ( 0 - $24 \mathrm{~h}$ ) expression analysis of Mj NOS was performed using pooled gill tissues from three individual shrimp that were injected with $V$. penaeicida. By semiquantitative analysis, the relative expression ratio of the Mj NOS was calculated.

\section{Results}

\section{Cloning and sequencing}

The entire cloned sequence of the Mj NOS cDNA is 4,616 bp with a 3,561 bp open reading frame coding for 1,187 (Fig. 1) amino acids. Additionally, it has an estimated mass of $134 \mathrm{kDa}$.

\section{Sequence homology and domain structure}

To determine the percent homology with other NOS genes, sequence alignment was performed. The sequence homologies of Mj NOS were as follows: $82.3 \%$ with land crab NOS (Gl NOS); $60.1 \%$ with cricket Gryllus bimaculatus NOS (Gb NOS); and $40.3 \%$ with rat nNOS (Table $2 \mathrm{~A}$ ). The Mj NOS amino acid sequence was aligned with the published NOS sequences of the land crab, mollusks (cuttlefish), chordates (i.e., amphioxus and sea squirt), insects (i.e., cricket, silkworm, fruit fly and honey bee) and rat (Fig. 2). Mj NOS consists of an oxygenase domain, CaM binding domain and a reductase domain with 372, 24 and 668 amino acids, respectively (Fig. 2). 


\begin{tabular}{|c|c|}
\hline MKEVNKPQRLQNISSGNEVYDSLHTRTQTEGVCTRHLCNGALMVPRKRGTEPRSRDEVLKLAKDFIDEYY & 70 \\
\hline QSIKRFNSEQHRQRWEQITREIDDRGTYDLTQTELVYGAKLGWRNS PRCIGRIQWSKLQVFDARYVTTAS & 140 \\
\hline GMFEALCNHIKYGTNKGDLRSAITIFPPRTDGKHDFRVWNSQLISYAGYKQEDGTIIGDPINVEFTEVCV & 210 \\
\hline RLGWKPKGGRWDVLPLVLSANGHDPEWFDI PQDLILTIPISHPEYKWFSDLDLQWYALPAVASLMFDCGG & 280 \\
\hline LECPAAPFNGWYMVSEIGTRDLCDPHLFNILKTVGRSMGLDTRS PTNLWKDKALVEVNIAVLHSFQSLNV & 350 \\
\hline TIVDHHSAAESFMKHFENEQRLRGGCPADWVWIVPPLSGSITPVFHQEMSLYYLKPSYEYQDPAWKTHVW & 420 \\
\hline KKTKDVNRNSVRKTKRKFRFKEIARAVKFTSKLFGKALSKRIKATILYATETGKSEMYAKKLGEIFGHTF & 490 \\
\hline NAQVCCMADYDLINIEHEALVLVVTSTFGNGDP PENGEEFAKNLYAMKVSGTAAGIDDVTSSMHRSLSFM & 560 \\
\hline RMNSLTEGAGAPSTPLENGLASCNLRGSITSDIMSEDNFGPLSNVRFAVFALGSSAYPNFCAFGKYVDNL & 630 \\
\hline LSELGGERLVKLTCGDELAGQEQAFKQWAGDVFTVGCETFCLDDDVAMKEATAALKIEATAALKIEATAS & 700 \\
\hline ANKIKLAPCTKTDGIDIGLSRMHGKRVRSCQVLASRNLHGENASRWTQQVILTTGGVNELNYSPGDHVAI & 770 \\
\hline LPANRKELIDAVLARLDNCPNPDEPIQVQVQKEVHSLNGVIQTWEPHERLPSTTVRELLTRYLDITTPPT & 840 \\
\hline PNFLHLLAEYAYDNDQRTRLDQLATDPHEYEEWKHLRYPHLKEVLEEFPSVVLDAGLLLTHLPLMGPRFY & 910 \\
\hline SISSSPDAHPGQIHITVAVVIYNTENGKGPLHYGVCSNYLKEVKAGNHIELFVRSASSFHMPRDPNVPII & 980 \\
\hline LVGPGTGVAPFRGFWHHRHYMLKHKKENAGKMTLFFGCRTRALDLYADEKEAMQRTGVLSQTYLALSREP & 1050 \\
\hline TIKKTYVQDLLVGVGSEVYRQVVEQKGHFYVCGDCTMAECVYQKLKS IVQEHGRLSDQEVENEMLQMRDE & 1120 \\
\hline NRYHEDIFGITLRTEEIHRQKRESARVKMSSISQAGPPTPPVTQAPTNFAQEAAA & \\
\hline
\end{tabular}

Fig. 1. The amino acid sequence of kuruma shrimp nitric oxide synthase (Mj NOS) cDNA $(4,616 \mathrm{bp})$. It contained a complete open reading frame (ORF) encoding a protein of 1,187 amino acids (residue number indicated on the right). GenBank accession \# AB485762.

Table 2. By the entire NOS or each domain, amino acid identity and similarity of the Mj NOS gene compared to other known NOS sequences. Upper triangle is identity. Lower triangle is similarity. A : entire NOS, B : CaM binding domain, C : oxygenase domain, D : reductase domain. The full name, abbreviation and accession number: M. japonicus NOS, Mj NOS and AB485762; Gecarcinus lateralis, Gl NOS and AY552549; Gryllus bimaculatus NOS, Gb NOS and AB477987; Bombyx mori NOS, Bm NOS and NM_001043498; Drosophila melanogaster NOS, Dm NOS and U25117; Apis mellifera NOS, Am NOS and NM_001012962; Sepia officinalis NOS, So NOS and AY582749; Branchiostoma floridae NOS, Bf NOS and AF396968; Ciona intestinalis NOS, Ci NOS and XM_002120231; Rattus norvegicus nNOS, Rn nNOS and X59949.1; Rattus norvegicus eNOS, Rn eNOS and NM_021838.2; Rattus norvegicus iNOS, Rn iNOS and NM_012611.3.

A

\begin{tabular}{|l|c|c|c||c|c|c||c||c|c|c||c|c|}
\hline Entire NOS & $\mathbf{1}$ & $\mathbf{2}$ & $\mathbf{3}$ & $\mathbf{4}$ & $\mathbf{5}$ & $\mathbf{6}$ & $\mathbf{7}$ & $\mathbf{8}$ & $\mathbf{9}$ & $\mathbf{1 0}$ & $\mathbf{1 1}$ & $\mathbf{1 2}$ \\
\hline 1. Mj NOS & & 82.3 & 60.1 & 49.7 & 47.3 & 58.2 & 48.5 & 40.6 & 38.6 & 40.3 & 45.7 & 41.9 \\
\hline 2. Gl NOS & $\mathbf{9 0 . 8}$ & & 59.4 & 49.2 & 46.8 & 57.0 & 48.7 & 40.7 & 38.2 & 40.2 & 45.8 & 42.3 \\
\hline $\mathbf{3 . ~ G b ~ N O S ~}$ & $\mathbf{7 6 . 0}$ & $\mathbf{7 5 . 1}$ & & 55.4 & 56.5 & 71.3 & 48.0 & 42.4 & 40.3 & 42.2 & 46.8 & 43.8 \\
\hline 4. Bm NOS & $\mathbf{6 8 . 8}$ & $\mathbf{6 8 . 2}$ & $\mathbf{7 1 . 5}$ & & 47.1 & 53.7 & 43.8 & 39.9 & 39.1 & 39.8 & 44.7 & 42.2 \\
\hline 5. Dm NOS & $\mathbf{6 3 . 6}$ & $\mathbf{6 3 . 6}$ & $\mathbf{6 9 . 6}$ & $\mathbf{6 3 . 3}$ & & 52.2 & 40.2 & 41.2 & 41.1 & 42.9 & 41.3 & 39.8 \\
\hline 6. Am NOS & $\mathbf{7 4 . 8}$ & $\mathbf{7 4 . 4}$ & $\mathbf{8 4 . 4}$ & $\mathbf{7 0 . 1}$ & $\mathbf{6 6 . 7}$ & & 48.0 & 40.6 & 39.7 & 40.1 & 46.8 & 44.0 \\
\hline 7. So NOS & $\mathbf{6 7 . 3}$ & $\mathbf{6 6 . 6}$ & $\mathbf{6 7 . 6}$ & $\mathbf{6 2 . 9}$ & $\mathbf{5 8 . 8}$ & $\mathbf{6 8 . 2}$ & & 44.5 & 38.6 & 42.0 & 45.4 & 42.5 \\
\hline 8. Bf NOS & $\mathbf{5 8 . 4}$ & $\mathbf{5 8 . 3}$ & $\mathbf{5 8 . 9}$ & $\mathbf{5 8 . 9}$ & $\mathbf{6 0 . 7}$ & $\mathbf{5 7 . 7}$ & $\mathbf{6 0 . 4}$ & & 44.6 & 46.8 & 42.9 & 41.8 \\
\hline 9. Ci NOS & $\mathbf{5 7 . 2}$ & $\mathbf{5 6 . 7}$ & $\mathbf{5 7 . 9}$ & $\mathbf{5 7 . 7}$ & $\mathbf{6 1 . 2}$ & $\mathbf{5 7 . 2}$ & $\mathbf{5 6 . 3}$ & $\mathbf{6 3 . 5}$ & & 48.4 & 42.7 & 41.1 \\
\hline 10. Rn nNOS & $\mathbf{5 7 . 5}$ & $\mathbf{5 6 . 3}$ & $\mathbf{5 8 . 9}$ & $\mathbf{5 7 . 5}$ & $\mathbf{6 1 . 0}$ & $\mathbf{5 6 . 2}$ & $\mathbf{5 6 . 8}$ & $\mathbf{6 4 . 0}$ & $\mathbf{6 7 . 0}$ & & 48.8 & 43.5 \\
\hline 11. Rn eNOS & $\mathbf{6 5 . 1}$ & $\mathbf{6 4 . 6}$ & $\mathbf{6 3 . 9}$ & $\mathbf{6 3 . 7}$ & $\mathbf{5 7 . 4}$ & $\mathbf{6 4 . 2}$ & $\mathbf{6 3 . 1}$ & $\mathbf{5 8 . 8}$ & $\mathbf{5 9 . 1}$ & $\mathbf{6 2 . 6}$ & & 48.5 \\
\hline 12. Rn iNOS & $\mathbf{6 1 . 4}$ & $\mathbf{6 0 . 2}$ & $\mathbf{6 2 . 8}$ & $\mathbf{6 1 . 1}$ & $\mathbf{5 5 . 8}$ & $\mathbf{6 2 . 1}$ & $\mathbf{6 3 . 6}$ & $\mathbf{5 8 . 0}$ & $\mathbf{5 7 . 7}$ & $\mathbf{5 7 . 9}$ & $\mathbf{6 5 . 1}$ & \\
\hline
\end{tabular}


Table 2. (contd.)

B

\begin{tabular}{|l|c|c||c|c|c||c|c|c||c|c|c|c|}
$\begin{array}{c}\text { CaM binding } \\
\text { domain }\end{array}$ & $\mathbf{1}$ & $\mathbf{2}$ & $\mathbf{3}$ & $\mathbf{4}$ & $\mathbf{5}$ & $\mathbf{6}$ & $\mathbf{7}$ & $\mathbf{8}$ & $\mathbf{9}$ & $\mathbf{1 0}$ & $\mathbf{1 1}$ & $\mathbf{1 2}$ \\
\hline 1. Mj NOS & & 95.8 & 79.2 & 75.0 & 79.2 & 79.2 & 62.5 & 33.3 & 45.8 & 50.0 & 45.8 & 37.5 \\
\hline 2. Gl NOS & $\mathbf{1 0 0}$ & & 75.0 & 75.0 & 75.0 & 75.0 & 66.7 & 33.3 & 45.8 & 45.8 & 41.7 & 41.7 \\
\hline 3. Gb NOS & $\mathbf{9 1 . 7}$ & $\mathbf{9 1 . 7}$ & & 87.5 & 95.8 & 100 & 62.5 & 33.3 & 41.7 & 54.2 & 41.7 & 33.3 \\
\hline 4. Bm NOS & $\mathbf{8 3 . 3}$ & $\mathbf{8 3 . 3}$ & $\mathbf{8 7 . 5}$ & & 83.3 & 87.5 & 58.3 & 29.2 & 37.5 & 50.0 & 37.5 & 29.2 \\
\hline 5. Dm NOS & $\mathbf{9 1 . 7}$ & $\mathbf{9 1 . 7}$ & $\mathbf{1 0 0}$ & $\mathbf{8 7 . 5}$ & & 95.8 & 62.5 & 33.3 & 37.5 & 54.2 & 41.7 & 33.3 \\
\hline 6. Am NOS & $\mathbf{9 1 . 7}$ & $\mathbf{9 1 . 7}$ & $\mathbf{1 0 0}$ & $\mathbf{8 7 . 5}$ & $\mathbf{1 0 0}$ & & 62.5 & 33.3 & 41.7 & 54.2 & 41.7 & 33.3 \\
\hline 7. So NOS & $\mathbf{8 3 . 3}$ & $\mathbf{8 3 . 3}$ & $\mathbf{8 3 . 3}$ & $\mathbf{7 0 . 8}$ & $\mathbf{8 3 . 3}$ & $\mathbf{8 3 . 3}$ & & 37.5 & 29.2 & 58.3 & 41.7 & 33.3 \\
\hline 8. Bf NOS & $\mathbf{5 0 . 0}$ & $\mathbf{5 0 . 0}$ & $\mathbf{5 8 . 3}$ & $\mathbf{5 4 . 2}$ & $\mathbf{6 2 . 5}$ & $\mathbf{5 8 . 3}$ & $\mathbf{6 6 . 7}$ & & 20.8 & 37.5 & 41.7 & 34.6 \\
\hline 9. Ci NOS & $\mathbf{6 6 . 7}$ & $\mathbf{6 6 . 7}$ & $\mathbf{7 0 . 8}$ & $\mathbf{6 6 . 7}$ & $\mathbf{7 0 . 8}$ & $\mathbf{7 0 . 8}$ & $\mathbf{6 6 . 7}$ & $\mathbf{4 5 . 8}$ & & 30.4 & 31.8 & 27.3 \\
\hline 10. Rn nNOS & $\mathbf{7 0 . 8}$ & $\mathbf{7 0 . 8}$ & $\mathbf{7 0 . 8}$ & $\mathbf{6 6 . 7}$ & $\mathbf{7 0 . 8}$ & $\mathbf{7 0 . 8}$ & $\mathbf{7 5 . 0}$ & $\mathbf{5 4 . 2}$ & $\mathbf{6 5 . 2}$ & & 52.2 & 43.5 \\
\hline 11. Rn eNOS & $\mathbf{6 2 . 5}$ & $\mathbf{6 2 . 5}$ & $\mathbf{5 8 . 3}$ & $\mathbf{5 4 . 2}$ & $\mathbf{5 8 . 3}$ & $\mathbf{5 8 . 3}$ & $\mathbf{5 8 . 3}$ & $\mathbf{5 8 . 3}$ & $\mathbf{6 3 . 6}$ & $\mathbf{6 0 . 9}$ & & 22.7 \\
\hline 12. Rn iNOS & $\mathbf{5 4 . 2}$ & $\mathbf{5 4 . 2}$ & $\mathbf{5 0 . 0}$ & $\mathbf{4 5 . 8}$ & $\mathbf{5 0 . 0}$ & $\mathbf{5 0 . 0}$ & $\mathbf{5 4 . 2}$ & $\mathbf{5 4 . 2}$ & $\mathbf{5 9 . 1}$ & $\mathbf{6 0 . 9}$ & $\mathbf{4 5 . 5}$ & \\
\hline
\end{tabular}

C

\begin{tabular}{|l|l||c|c||c|c|c||c|c|c||c|c|c|}
\hline $\begin{array}{c}\text { Oxygenase } \\
\text { doamin }\end{array}$ & $\mathbf{1}$ & $\mathbf{2}$ & $\mathbf{3}$ & $\mathbf{4}$ & $\mathbf{5}$ & $\mathbf{6}$ & $\mathbf{7}$ & $\mathbf{8}$ & $\mathbf{9}$ & $\mathbf{1 0}$ & $\mathbf{1 1}$ & $\mathbf{1 2}$ \\
\hline 1. Mj NOS & & 89.0 & 73.7 & 64.0 & 70.8 & 71.5 & 66.6 & 61.8 & 57.7 & 65.2 & 58.8 & 61.2 \\
\hline 2. Gl NOS & $\mathbf{9 6 . 0}$ & & 73.7 & 66.1 & 70.2 & 71.8 & 68.4 & 63.1 & 58.2 & 66.0 & 62.2 & 62.8 \\
\hline 3. Gb NOS & $\mathbf{8 6 . 3}$ & $\mathbf{8 5 . 8}$ & & 67.3 & 75.6 & 81.5 & 65.2 & 61.8 & 56.5 & 64.6 & 61.7 & 60.6 \\
\hline 4. Bm NOS & $\mathbf{8 1 . 2}$ & $\mathbf{8 0 . 4}$ & $\mathbf{8 3 . 1}$ & & 63.5 & 68.3 & 63.4 & 61.6 & 56.3 & 64.9 & 58.2 & 60.4 \\
\hline 5. Dm NOS & $\mathbf{8 5 . 8}$ & $\mathbf{8 5 . 8}$ & $\mathbf{8 7 . 7}$ & $\mathbf{8 1 . 8}$ & & 73.7 & 62.6 & 59.6 & 55.8 & 64.9 & 59.3 & 59.0 \\
\hline 6. Am NOS & $\mathbf{8 7 . 1}$ & $\mathbf{8 7 . 4}$ & $\mathbf{9 2 . 2}$ & $\mathbf{8 2 . 5}$ & $\mathbf{8 7 . 1}$ & & 66.0 & 61.0 & 59.3 & 64.6 & 60.4 & 62.5 \\
\hline 7. So NOS & $\mathbf{8 4 . 0}$ & $\mathbf{8 4 . 2}$ & $\mathbf{8 1 . 6}$ & $\mathbf{7 8 . 6}$ & $\mathbf{8 1 . 3}$ & $\mathbf{8 1 . 6}$ & & 65.5 & 57.8 & 63.8 & 59.6 & 64.1 \\
\hline 8. Bf NOS & $\mathbf{8 1 . 2}$ & $\mathbf{8 1 . 0}$ & $\mathbf{7 8 . 3}$ & $\mathbf{7 9 . 4}$ & $\mathbf{7 8 . 6}$ & $\mathbf{7 8 . 0}$ & $\mathbf{8 1 . 3}$ & & 54.4 & 60.1 & 54.8 & 59.3 \\
\hline 9. Ci NOS & $\mathbf{7 7 . 2}$ & $\mathbf{7 6 . 7}$ & $\mathbf{7 6 . 1}$ & $\mathbf{7 4 . 8}$ & $\mathbf{7 5 . 1}$ & $\mathbf{7 6 . 7}$ & $\mathbf{7 7 . 7}$ & $\mathbf{7 3 . 5}$ & & 60.4 & 59.0 & 59.8 \\
\hline 10. Rn nNOS & $\mathbf{7 9 . 5}$ & $\mathbf{8 0 . 3}$ & $\mathbf{8 0 . 1}$ & $\mathbf{7 9 . 8}$ & $\mathbf{7 9 . 5}$ & $\mathbf{8 0 . 9}$ & $\mathbf{8 0 . 1}$ & $\mathbf{7 6 . 6}$ & $\mathbf{7 8 . 5}$ & & 69.1 & 66.8 \\
\hline 11. Rn eNOS & $\mathbf{7 6 . 1}$ & $\mathbf{7 6 . 1}$ & $\mathbf{7 4 . 7}$ & $\mathbf{7 3 . 1}$ & $\mathbf{7 4 . 7}$ & $\mathbf{7 5 . 3}$ & $\mathbf{7 5 . 3}$ & $\mathbf{7 0 . 7}$ & $\mathbf{7 4 . 8}$ & $\mathbf{8 2 . 4}$ & & 64.4 \\
\hline 12. Rn iNOS & $\mathbf{7 8 . 2}$ & $\mathbf{7 8 . 5}$ & $\mathbf{7 7 . 1}$ & $\mathbf{7 6 . 1}$ & $\mathbf{7 5 . 3}$ & $\mathbf{7 6 . 6}$ & $\mathbf{7 7 . 9}$ & $\mathbf{7 6 . 3}$ & $\mathbf{7 5 . 6}$ & $\mathbf{8 2 . 2}$ & $\mathbf{7 7 . 7}$ & \\
\hline \hline
\end{tabular}


Table 2. (contd.)

\begin{tabular}{|l|c|c|c||c|c|c|c||c|c|c|c|c|}
\hline $\begin{array}{c}\text { Reductase } \\
\text { domain }\end{array}$ & $\mathbf{1}$ & $\mathbf{2}$ & $\mathbf{3}$ & $\mathbf{4}$ & $\mathbf{5}$ & $\mathbf{6}$ & $\mathbf{7}$ & $\mathbf{8}$ & $\mathbf{9}$ & $\mathbf{1 0}$ & $\mathbf{1 1}$ & $\mathbf{1 2}$ \\
\hline 1. Mj NOS & & 83.0 & 59.6 & 49.3 & 50.9 & 55.9 & 43.7 & 42.4 & 43.0 & 45.7 & 45.1 & 40.0 \\
\hline 2. Gl NOS & $\mathbf{9 1 . 5}$ & & 59.3 & 48.0 & 51.0 & 54.7 & 43.6 & 42.6 & 42.7 & 45.5 & 44.3 & 40.1 \\
\hline 3. Gb NOS & $\mathbf{7 4 . 9}$ & $\mathbf{7 4 . 7}$ & & 53.1 & 61.6 & 68.2 & 43.6 & 43.5 & 44.0 & 46.9 & 45.6 & 41.3 \\
\hline $\mathbf{4 . ~ B m ~ N O S ~}$ & $\mathbf{6 8 . 1}$ & $\mathbf{6 7 . 6}$ & $\mathbf{7 0 . 0}$ & & 48.6 & 49.8 & 40.1 & 37.7 & 40.7 & 40.7 & 42.9 & 36.9 \\
\hline $\mathbf{5 . ~ D m ~ N O S ~}$ & $\mathbf{6 8 . 9}$ & $\mathbf{6 9 . 3}$ & $\mathbf{7 7 . 8}$ & $\mathbf{6 7 . 1}$ & & 55.0 & 41.5 & 42.7 & 42.6 & 43.8 & 42.5 & 40.3 \\
\hline $\mathbf{6 .}$ Am NOS & $\mathbf{7 2 . 3}$ & $\mathbf{7 2 . 7}$ & $\mathbf{8 4 . 4}$ & $\mathbf{6 9 . 1}$ & $\mathbf{7 4 . 3}$ & & 41.4 & 41.7 & 42.5 & 44.4 & 46.4 & 40.2 \\
\hline 7. So NOS & $\mathbf{6 3 . 9}$ & $\mathbf{6 3 . 4}$ & $\mathbf{6 4 . 9}$ & $\mathbf{6 1 . 8}$ & $\mathbf{6 4 . 5}$ & $\mathbf{6 3 . 7}$ & & 48.8 & 43.8 & 50.9 & 45.4 & 39.4 \\
\hline $\mathbf{8 . ~ B f ~ N O S ~}$ & $\mathbf{6 0 . 3}$ & $\mathbf{6 1 . 0}$ & $\mathbf{6 3 . 4}$ & $\mathbf{5 8 . 8}$ & $\mathbf{6 2 . 9}$ & $\mathbf{6 2 . 9}$ & $\mathbf{6 7 . 7}$ & & 48.8 & 51.1 & 46.9 & 42.3 \\
\hline $\mathbf{9 . ~ C i ~ N O S ~}$ & $\mathbf{6 2 . 7}$ & $\mathbf{6 2 . 4}$ & $\mathbf{6 5 . 5}$ & $\mathbf{6 2 . 0}$ & $\mathbf{6 3 . 9}$ & $\mathbf{6 4 . 9}$ & $\mathbf{6 4 . 8}$ & $\mathbf{6 7 . 0}$ & & 52.1 & 47.4 & 42.5 \\
\hline $\mathbf{1 0 .}$ Rn nNOS & $\mathbf{6 6 . 0}$ & $\mathbf{6 4 . 5}$ & $\mathbf{6 8 . 8}$ & $\mathbf{6 2 . 0}$ & $\mathbf{6 4 . 4}$ & $\mathbf{6 5 . 3}$ & $\mathbf{6 8 . 8}$ & $\mathbf{6 9 . 4}$ & $\mathbf{7 2 . 5}$ & & 56.3 & 46.7 \\
\hline $\mathbf{1 1 . ~ R n ~ e N O S ~}$ & $\mathbf{6 2 . 7}$ & $\mathbf{6 3 . 0}$ & $\mathbf{6 4 . 0}$ & $\mathbf{6 1 . 1}$ & $\mathbf{6 1 . 2}$ & $\mathbf{6 4 . 9}$ & $\mathbf{6 5 . 1}$ & $\mathbf{6 6 . 2}$ & $\mathbf{6 6 . 8}$ & $\mathbf{7 4 . 2}$ & & 46.9 \\
\hline $\mathbf{1 2 .}$ Rn iNOS & $\mathbf{5 6 . 3}$ & $\mathbf{5 5 . 6}$ & $\mathbf{5 7 . 6}$ & $\mathbf{5 6 . 3}$ & $\mathbf{5 7 . 1}$ & $\mathbf{5 6 . 6}$ & $\mathbf{6 0 . 0}$ & $\mathbf{6 0 . 8}$ & $\mathbf{6 2 . 8}$ & $\mathbf{6 5 . 1}$ & $\mathbf{6 4 . 8}$ & \\
\hline \hline
\end{tabular}

The heme binding and tetrahydrobiopterin $\left(\mathrm{BH}_{4}\right)$ domains were well-conserved (77.3-95.5\% and 72.7\%-100\%) in the oxygenase domain (Table 3). In terms of the CaM binding domain, Mj NOS demonstrated the highest homology with Gl NOS $(95.8 \%)$ followed by insect NOSs (75.0-79.2\%) (Table 2B) The CaM binding domain of $\mathrm{Mj}$ NOS showed a lower homology than those of amphioxus NOS and rat iNOS (Table 2B). In both the oxygenase and reductase domains, Mj NOS is found to have the highest homology with G1 NOS at levels of $89.0 \%$ and $83.0 \%$, respectively; however, in the oxygenase and reductase domains, when the Mj NOS gene was compared with other NOS genes, it showed 57.7-73.7\% and 40.0-59.6\% homologies, respectively (Tables 2C and 2D).

To determine the similarity among them, the complete domain structure of $\mathrm{Mj}$ NOS was compared with those of other NOSs (Fig. 3). Regarding the domain structure, the reductase domain of Mj NOS contained conserved binding motifs for FMN, FAD and NADPH which are typical structures of NOS. When compared among species, the arrangements of those domains were found to be common (Fig. 3). However, Mj NOS does not has the glutamine-rich sequence that has been observed in the N-terminal region of Dm NOS, the PDZ domain in chordate NOSs (i.e., Bf and Ci NOSs) and rat nNOS. 


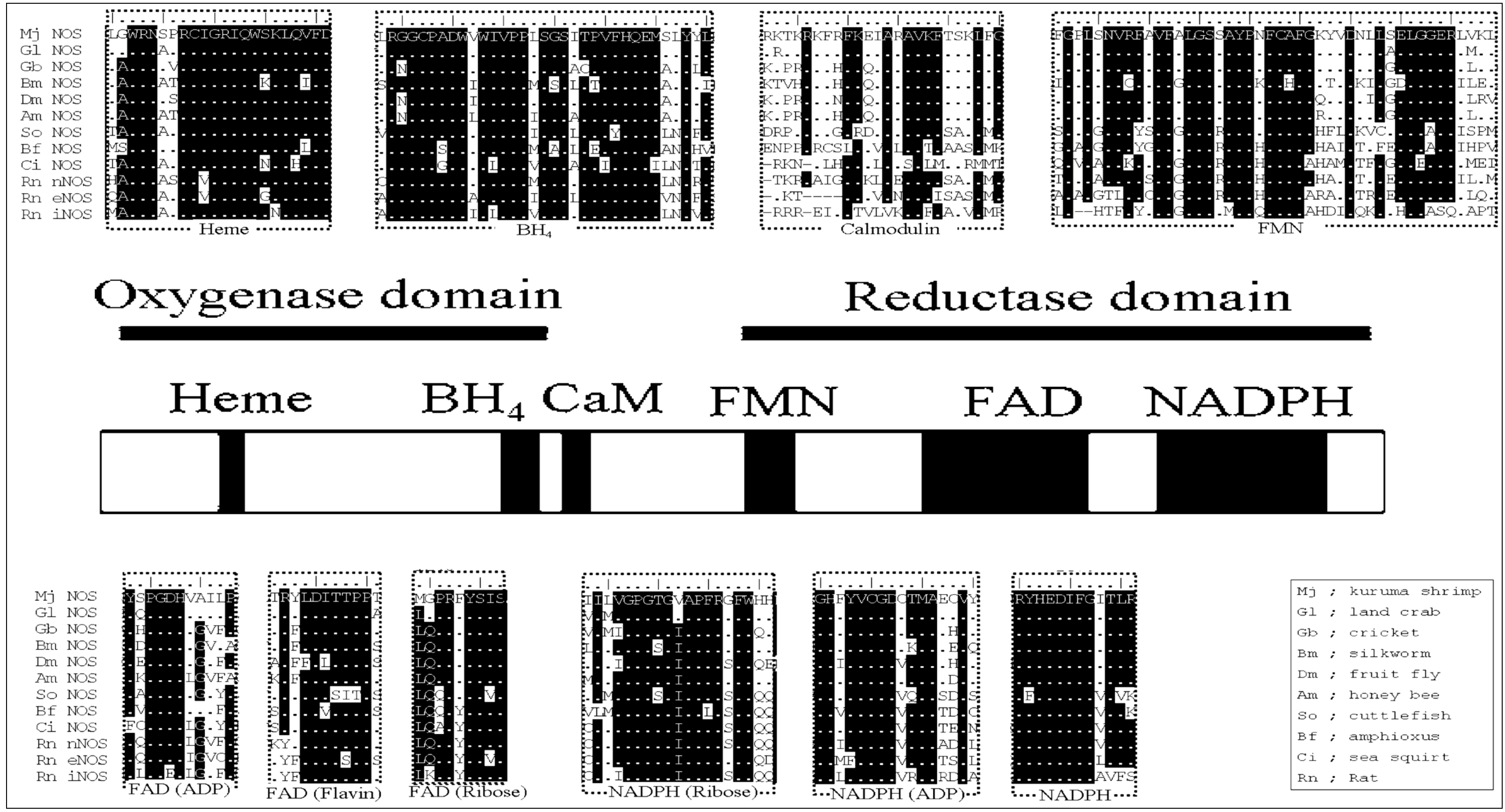

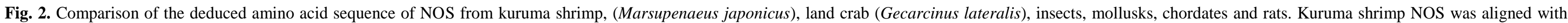

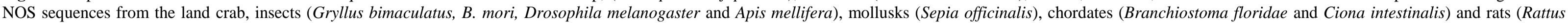

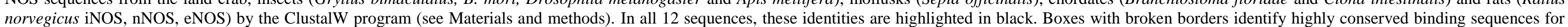

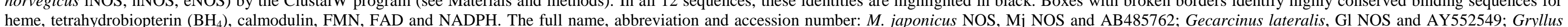

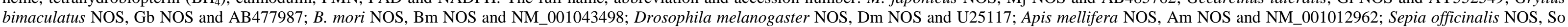

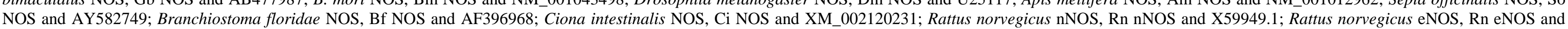
NM_021838.2; Rattus norvegicus iNOS, Rn iNOS and NM_012611.3. 
Table 3. In the heme, $\mathrm{BH}_{4}$ in the oxygenase domain and FMN, FAD and NADPH in the reductase domain, amino acid identity in the MjNOS gene compared to other known NOS sequences.

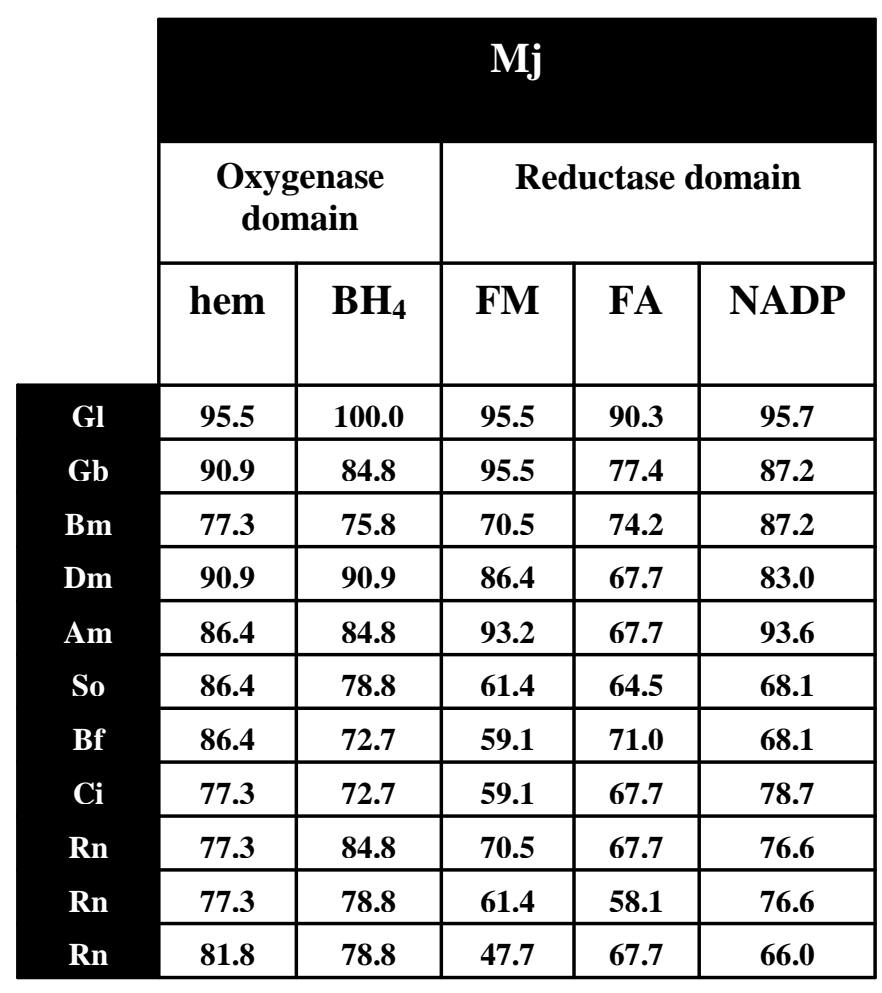




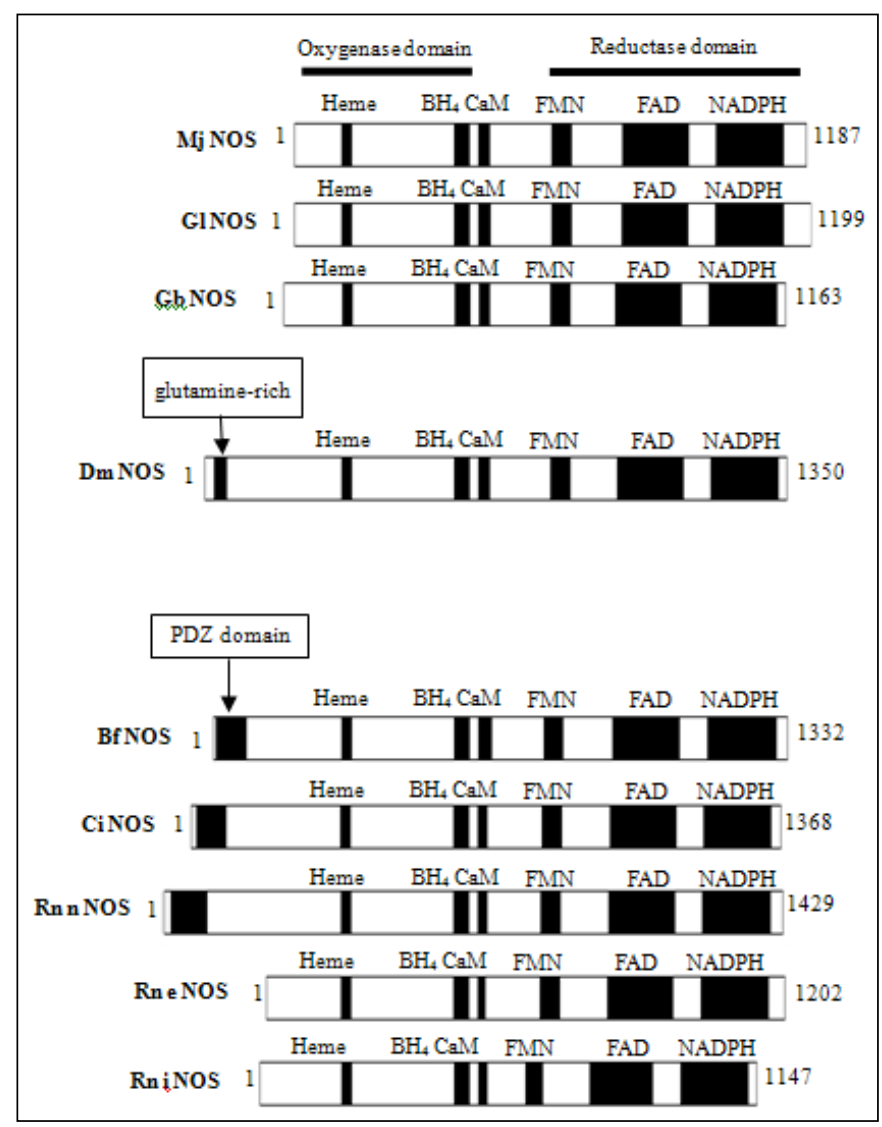

Fig. 3. Domain organization of NOS from the animals shown in Fig. 2. The ORFs from $M$. japonicus NOS (Mj NOS) and other NOS are compared. The oxygenase domain contains hemebinding and tetrahydrobiopterin $\left(\mathrm{BH}_{4}\right)$ domains. The reductase domain contains binding domains for FMN, FAD and NADPH. A calmodulin $(\mathrm{CaM})$ binding domain is located between the oxygenase and reductase domains and is involved in dimerization and regulation of catalytic activity (Regulski \& Tully 1995 and Stasiv et al. 2001). Near the amino terminus, D. melanogaster NOS (Dm NOS) has a Gln-rich sequence. B. floridae NOS (Bf NOS), $C$. intestinalis NOS (Ci NOS) and rat Rn nNOS have a PDZ domain. The full name, abbreviation and accession number: M. japonicus NOS, Mj NOS and AB485762; Gecarcinus lateralis, Gl NOS and AY552549; Gryllus bimaculatus NOS, Gb NOS and AB477987; Drosophila melanogaster NOS, Dm NOS and U25117; Branchiostoma floridae NOS, Bf NOS and AF396968; Ciona intestinalis NOS, Ci NOS and XM_002120231; Rattus norvegicus nNOS, Rn nNOS and X59949.1; Rattus norvegicus eNOS, Rn eNOS and NM_021838.2; Rattus norvegicus iNOS, Rn iNOS and NM_012611.3.

\section{Phylogenetic analysis}

Known NOS sequences were compared using sequence alignments to perform a phylogenetic analysis. The data showed that Mj NOS was closely related to Gl NOS, and that the NOSs of insects of major taxonomic groups including Orthoptera $(G$. 
bimaculatus), Hymenoptera (A. mellifera), Diptera (D. melanogaster) and Lepidoptera (B. mori) formed a cluster. Mollusks ( $S$. officinalis), chordates (B. floridae and $C$. intestinalis) and vertebrate NOSs formed another distinct cluster. Within the vertebrates, the inducible NOS (iNOS) and noninducible NOSs (i.e., nNOS and eNOS) were in separate clusters (Fig. 4).

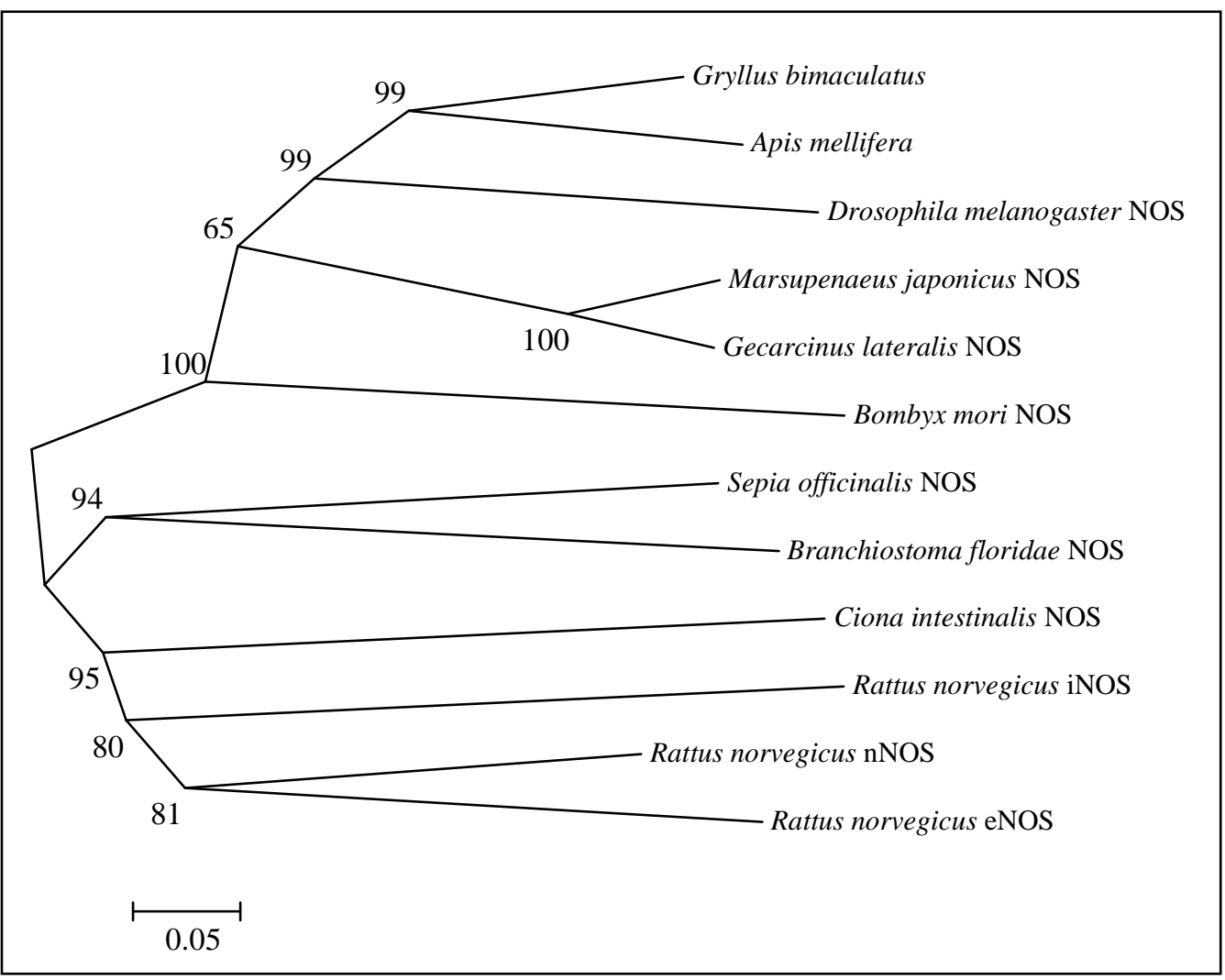

Fig. 4. Phylogenetic relationships of NO synthases from crustacean, insects, mollusks, chordates and mammals. By the ClustalW and Tree view programs, the deduced amino acid sequences were analyzed. Crustacean NOS sequences form a group divergent from molluscan and mammalian NOS sequences. The land crab NOS diverged from the insects within the arthropods. Accession numbers are the same as those given in the legend for Fig. 2.

\section{Expression analysis}

A marked increase was observed in the Mj NOS expression level in the gill, heart, lymphoid organ and thoracic ganglion tissues following $V$. penaeicida injection, whereas a decrease was observed in the testis (Fig. 5). The relative expression level of $\mathrm{Mj}$ NOS in the gill of the $V$. penaeicida-injected group began increasing $1 \mathrm{~h}$ post $V$. penaeicida injection and peaked $12 \mathrm{~h}$ after; it then decreased $24 \mathrm{~h}$ after the injection in a time course experiment on Mj NOS expression (Fig. 6). 


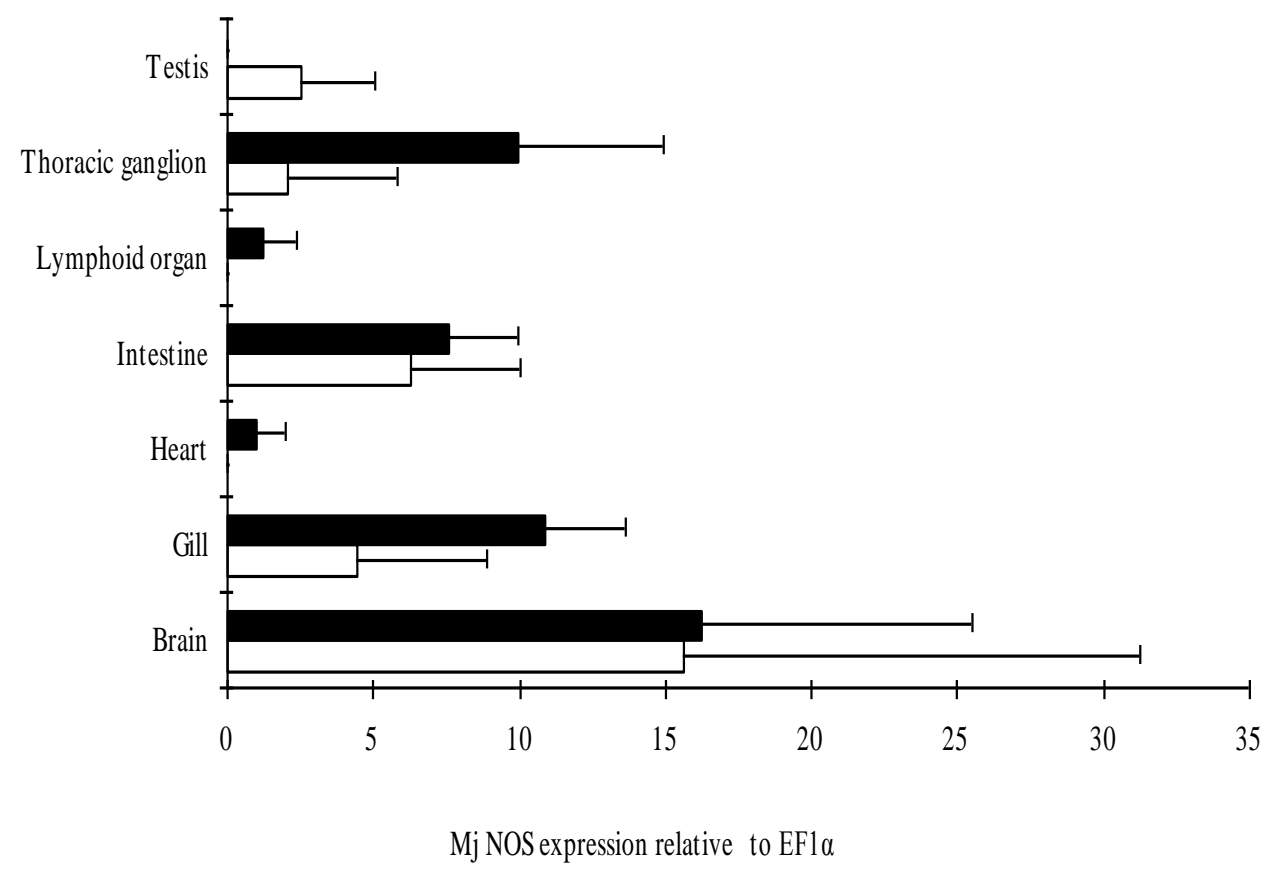

Fig. 5. The expression of Mj NOS from shrimp organs after injection of $V$. penaeicida. The expression ratio of Mj NOS was based on the quantity of expression of Mj NOS and EF1 $\alpha$. It was used a semi-quantitative analysis in the organs of kuruma shrimp. As a control, PBS injection was used.

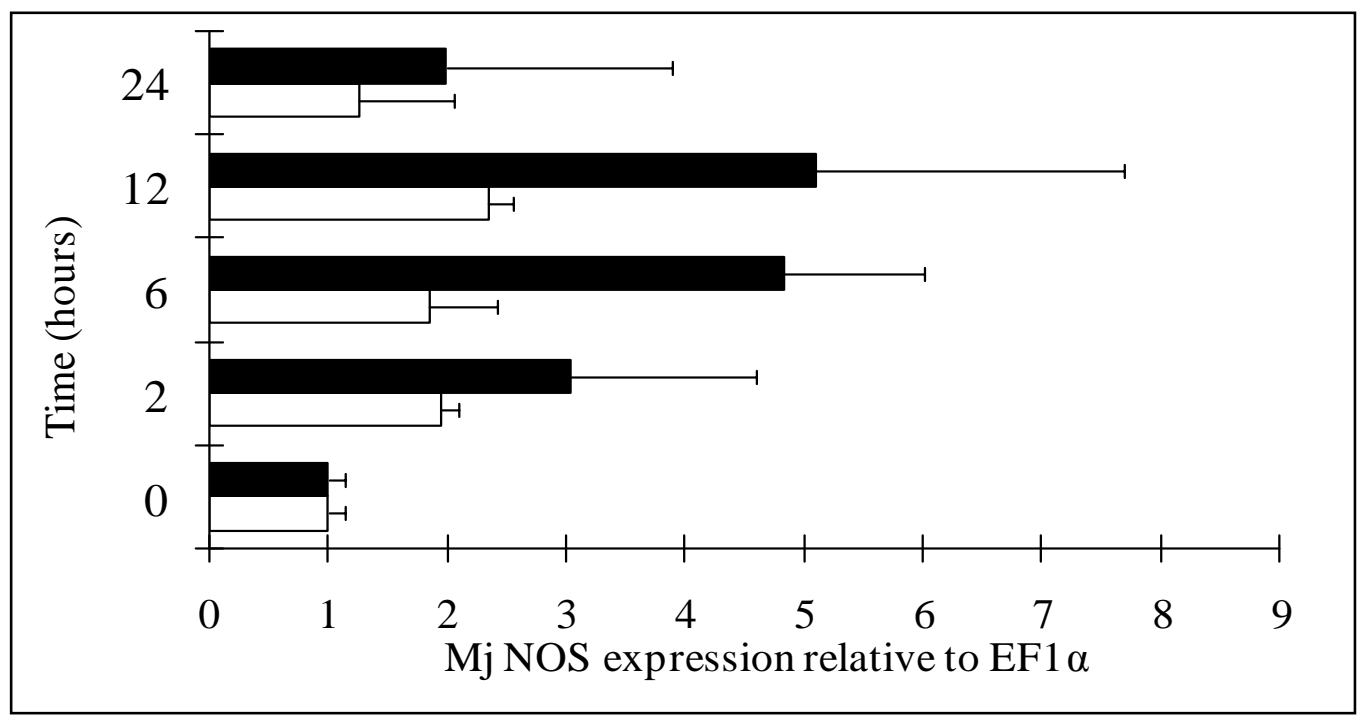

Fig. 6. Expression analyses of Mj NOS in kuruma shrimp gill after $V$. penaeicida injection. By a semi-quantitative analysis, the expression ratio of Mj NOS was analyzed over time $(0-24 \mathrm{~h})$ based on the quantity of expression of Mj NOS and EF1 $\alpha$. 


\section{Discussion}

The cDNA of the NOS gene of the kuruma shrimp (Mj NOS) from gill tissue mRNA was cloned. The ORF encoded a 1,187 amino acid protein with an estimated mass of $134 \mathrm{kDa}$. The molecular mass of Mj NOS was similar to that of land crab and crayfish NOSs (Lee et al. 2000) at $\sim 136$ and $\sim 138 \mathrm{kDa}$, respectively and showed the highest identity with the land crab NOS (82.3\%) and insect NOS (47.3-60.1\%). The highest identity of Mj NOS with land crab (82.3\%) shows that our original prediction of the high conservation of NOS in crustaceans is correct. The Mj NOS oxygenase domain showed an $89.0 \%$ sequence homology with Gl NOS and a $64.0-73.7 \%$ sequence homology with insect NOS. The Mj NOS reductase domain showed the highest (83.0\%) sequence homology with Gl NOS followed by the insect NOS (49.3-59.6\%). The $\mathrm{Mj}$ and Gl NOSs CaM binding domains showed 75.0-79.2\% homologies with those of insect NOSs, particularly those with similar amino acid sequences: $(\mathrm{KF}(\mathrm{R} / \mathrm{H} / \mathrm{N}) \mathrm{FK}(\mathrm{E} / \mathrm{Q}) \mathrm{IARAVKFTSKLFG})$. The amino acid sequence variation shown above is found to involve arginine (R) and glutamic acid (E) in crustacean NOSs and histidine $(\mathrm{H})$ or asparagine $(\mathrm{N})$ and glutamine $(\mathrm{Q})$ in insect NOSs. Conversely, the CaM binding domains of amphioxus NOS and rat iNOS show lower similarities (33.3 and $37.5 \%$ ) than those of crustacean NOSs. Among animal species, this suggests the CaM domain has higher variations than the oxygenase and reductase domains.

Drosophila NOS has 24 glutamine residues at its N-terminal end and this segment is a glutamine-rich domain that is involved in protein-protein interactions for the regulation of transcription activation (Regulski and Tully 1995; Yeh et al. 2006). However, no glutamine-rich domain was observed in Mj NOS, Gl NOS or other insect NOSs, except Dm NOS.

In the $\mathrm{N}$-terminal end of amphioxus $\mathrm{Bf}$, sea squirt $\mathrm{Cl}$ and rat $\mathrm{nNOSs}$, analysis of secondary structures showed a PDZ domain. In the rat, the interaction of PDZcontaining domains mediated the synaptic association of nNOS. It plays a central role in the formation of macromolecular signaling complexes (Brenman et al. 1996) which may function differently from amphioxus NOS and rat nNOS since Mj NOS and other invertebrate NOSs have no PDZ domain.

Phylogenetic analysis showed that the NOSs studied are divided into two distinct clusters. One cluster includes crustacean and insect NOSs and the other cluster includes mollusk, chordate and vertebrate NOSs.

After V. penaeicida injection in the gills, heart, lymphoid organ and thoracic ganglion of the kuruma shrimp, we observed the up-regulation of Mj NOS expression. 
However, in heart or lymphoid organs of the control (PBS-injected) shrimp, there was no Mj NOS expression. No Mj NOS expression was observed in the muscle or hepatopancreas of the injected or control group. In the crab tissues, no Gl NOS expression was observed (Kim et al. 2004). The NOS gene was detected in the ovaries of the crab, but not in either of the injected groups of the shrimp. In contrast, in the thoracic ganglion, the shrimp NOS gene was found, but the crab NOS gene was not. Interestingly, in the testis, the crab and control shrimp NOS genes were detected, but the NOS gene was not detected in the V. penaeicida-injected shrimp, suggesting the down-regulation of NOS expression. In the heart, no NOS genes were detected, except in the $V$. penaeicida-injected shrimp, suggesting the up-regulation of NOS expression after $V$. penaeicida injection. In the expression of the NOS gene, these differences between the shrimp and the crab may be due to differences in the species, molting cycle and the capability of up- or down-regulation by stimulation, as observed in insect NOS genes (Imamura et al. 2002). In shrimp hemocytes, none Mj NOS was found. However, the NOS activity has been reported in kuruma shrimp hemocytes following white spot syndrome virus infection (Jiang et al. 2006). In hemocytes, the non-detection of $\mathrm{Mj}$ NOS is considered off-target sampling timing of shrimp. Shrimp hemocytes were sampled $6 \mathrm{~h}$ after $V$. penaeicida injection. Mj NOS expression could be found 1 and $2 \mathrm{~h}$ after $V$. penaeicida injection. Additionally, NO production was also confirmed $4 \mathrm{~h}$ after the injection of LPS which was extracted from $V$. penaeicida in unpublished data.

The $\mathrm{Mj}$ NOS expression level began increasing $1 \mathrm{~h}$ after injection and then gradually peaked after $12 \mathrm{~h}$ after, determining the changes in Mj NOS expression level in the gill after $V$. penaeicida injection. It then decreased down to the normal level $24 \mathrm{~h}$ after injection. The $V$. penaeicida-injected group showed a higher expression level than the control group during the entire period. These results indicate that Mj NOS is clearly inducible using a $V$. penaeicida injection. NOS is also inducible after LPS injection in insects (Imamura et al. 2002). This inducible NOS has been demonstrated to be common in arthropods (Yeh et al. 2006 and Imamura et al. 2002). Further study is necessary to clarify the function and structure of inducible NOSs that may differ between various organs after transcription.

In the lungs of rats, LPS stimulation induced a large effect on iNOS gene expression with dose dependence. However, it induced a small effect on eNOS and nNOS gene expressions (McCluskie et al. 2004). In mammals, nNOS and eNOS are $\mathrm{Ca}^{2+} / \mathrm{CaM}$-dependent; however, iNOS is $\mathrm{Ca}^{2+}$-independent. NOS only has the $\mathrm{Ca}^{2+} / \mathrm{CaM}$-dependent form (Davies 2000; Korneev et al. 1998; Luckhart and Rosenberg 1999) similar to nNOS or eNOS in the system of enzyme activation by CaM in invertebrates. However, in crayfish hemocytes, $\mathrm{Ca}^{2+}$-independent LPS-inducible NOS 
activity has been reported. This inducible NO activity promotes bacterial adhesion and enhances bactericidal activity (Yeh et al. 2006).

Mj NOS had a higher similarity to mammalian nNOS than to iNOS or eNOS as determined on the basis of sequence and phylogenetic analyses in this study. However, Mj NOS was induced by $V$. penaeicida injection, suggesting that it is an inducible NOS after stimulation and has a higher similarity to mammalian iNOS in function. These contradictory observations suggest that nNOS may be the ancestor of eNOS and iNOS. More studies are required to better understand the molecular function and structure of $\mathrm{Mj}$ NOS in shrimp. The presence of NOS in crustacean non-neuronal tissues suggests that NO signaling is involved in physiological processes in addition to neuromodulation. In gonadal tissue, NOS may regulate gametogenesis and/or steroidogenesis (Kim et al. 2004).

\section{Acknowledgements}

This study was supported, in-part, by the University of Miyazaki's Program for the Support of Women in the Sciences and research grants from the Development Program for New Bio-industry Initiatives. This work was supported in-part by a grantin-aid from the Japan Society for the Promotion of Science.

\section{References}

Abu-Soud, H.M. and D.J. Stuehr. 1993. Nitric oxide synthases reveal a role for calmodulin in controlling electron transfer. Proceedings of the National Academy of Sciences of the United States of America 90:10769-10772.

Abu-Soud, H.M, L.L.Yoho and D.J. Stuehr. 1994. Calmodulin controls neuronal nitric-oxide synthase by a dual mechanism. Activation of intra- and interdomain electron transfer. Journal of Biological Chemistry 269:32047-32050.

Aktan, F. 2004. iNOS-mediated nitric oxide production and its regulation. Life Sciences 75:639-653.

Andrew, P.J. and B. Mayer. 1999. Enzymatic function of nitric oxide synthases. Cardiovascular Research 43:521-531.

Beck, G., T. Ellis, H. Zhang, W. Lin, K. Beauregard, G.S. Habicht and N. Truong. 2001. Nitric oxide production by coelomocytes of Asterias forbesi. Developmental and Comparative Immunology 25:1-10.

Bogdan, C., M. Röllinghoff and A. Diefenbach. 2000. The role of nitric oxide in innate immunity. Immunological Reviews 173:17-26.

Brenman, J.E., D.S. Chao, S.H. Gee, A.W. McGee, S.E. Craven, D.R. Santillano, Z. Wu, F. Huang, H. Xia, M.F. Peters, S.C. Froehner and D.S. Bredt. 1996. Interaction of nitric oxide synthase with the postsynaptic density protein PSD-95 and alpha1-syntrophin mediated by PDZ domains. Cell 84:757-767. 
Davies, S.A. 2000. Nitric oxide signalling in insects. Insect Biochemistry and Molecular Biology 30:1123-1138.

Fang F.C. 1997. Mechanisms of nitric oxide-related antimicrobial activity. Journal of Clinical Investigation 99:2818-2825.

Furchgott, R.F. 1990. The 1989 Ulf von Euler Lecture. Studies on endothelium-dependent vasodilation and the endothelium-derived relaxing factor. Acta Physiologica Scandinavica 139:257-270.

Gibbs, S.M. and J.W. Truman. 1998. Nitric oxide and cyclic GMP regulate retinal patterning in the optic lobe of Drosophila. Neuron 20:83-93.

Hall, T.A. 1999. BioEdit: A user-friendly biological sequence alignment editor and analysis program for Windows 95/98/NT. Nucleic Acids Symposium Series 41:95-98.

Imamura, M., J. Yang and M. Yamakawa. 2002. cDNA cloning, characterization and gene expression of nitric oxide synthase from the silkworm, Bombyx mori. Insect Molecular Biology 11:257-265.

Jiang, G., R. Yu and M. Zhou. 2006. Studies on nitric oxide synthase activity in haemocytes of shrimps Fenneropenaeus chinensis and Marsupenaeus japonicus after white spot syndrome virus infection. Nitric Oxide - Biology and Chemistry 14:219-227.

Kim, H.W., L.A. Batista, J.L. Hoppes, K.J. Lee and D.L. Mykles. 2004. A crustacean nitric oxide synthase expressed in nerve ganglia, Y-organ, gill and gonad of the tropical land crab, Gecarcinus lateralis. Journal of Experimental Biology 207:2845-2857.

Korneev, S. A., M.R. Piper, J. Picot, R. Phillips, E.I. Korneeva and M. O'Shea. 1998. Molecular characterization of NOS in a mollusc: expression in a giant modulatory neuron. Journal of Neurobiology 35:65-76.

Lee, C.Y., H.S. Zou, S.M. Yau,Y.R. Ju and C.S. Liau. 2000. Nitric oxide synthase activity and immunoreactivity in the crayfish Procambarus clarkii. NeuroReport 11:1273-1276.

Luckhart, S. and R. Rosenberg. 1999. Gene structure and polymorphism of an invertebrate nitric oxide synthase gene. Gene 232:25-34.

Luckhart, S., Y. Vodovotz, L. Ciu and R. Rosenberg. 1998. The mosquito Anopheles stephensi limits malaria parasite development with inducible synthesis of nitric oxide. Proceedings of the National Academy of Sciences of the United States of America 95:5700-5705.

McCluskie, K., M.A. Birrell, S. Wong and M.G. Belvisi. 2004. Nitric oxide as a noninvasive biomarker of lipopolysaccharide-induced airway inflammation:possible role in lung neutrophilia. Journal of Pharmacology and Experimental Therapeutics 311:625-633.

Momoyama, K. and K. Muroga. 2005. Diseases of cultured kuruma shrimp in Japan: a review. Fish Pathology 40:1-14.

Müller, U. 1996. Inhibition of nitric oxide synthase impairs a distinct form of long-term memory in the honeybee, Apis mellifera. Neuron 16:541-549.

Nappi, A.J., E. Vass, F. Frey and Y. Carton. 2000. Nitric oxide involvement in Drosophila immunity. Nitric Oxide - Biology and Chemistry 4:423-430.

Nathan, C. 1992. Nitric oxide as a secretory product of mammalian cells. FASEB Journal 6:3051-3064.

Nathan, C.F. and J.B. Hibbs Jr. 1991. Role of nitric oxide synthesis in macrophage antimicrobial activity. Current Opinion in Immunology 3:65-70.

Nighorn, A., N.J. Gibson, D.M. Rivers, J.G. Hildebrand and D.B. Morton. 1998. The nitric oxide-cGMP pathway may mediate communication between sensory afferents and 
projection neurons in the antennal lobe of Manduca sexta. Journal of Neuroscience 18:7244-7255.

Nishida, K., D.G. Harrison, J.P. Navas, A.A. Fisher, S.P. Dockery, M. Uematsu, R.M. Nerem, R.W. Alexander and T.J. Murphy. 1992. Molecular cloning and characterization of the constitutive bovine aortic endothelial cell nitric oxide synthase. Journal of Clinical Investigation 90:2092-2096.

O'Dell, T.J., R.D. Hawkins, E.R. Kandel and O. Arancio. 1991. Tests of the roles of two diffusible substances in long-term potentiation: Evidence for nitric oxide as a possible early retrograde messenger. Proceedings of the National Academy of Sciences of the United States of America 88:11285-11289.

Palmer, R.M.J., A.G. Ferrige and S. Moncada. 1987. Nitric oxide release accounts for the biological activity of endothelium-derived relaxing factor. Nature 327:524-526.

Regulski, M. and T. Tully. 1995. Molecular and biochemical characterization of dNOS: a Drosophila $\mathrm{Ca}^{2+} /$ calmodulin-dependent nitric oxide synthase. Proceedings of the National Academy of Sciences of the United States of America 92:9072-9076.

Schmidt, H.H.H.W., G.D. Gagne, M. Nakane, J.S. Pollock, M.F. Miller and F. Murad. 1992. Mapping of neural nitric oxide synthase in the rat suggests frequent co- localization with NADPH diaphorase but not with soluble guanylyl cyclase, and novel paraneural functions for nitrinergic signal transduction. Journal of Histochemistry and Cytochemistry 40:1439-1456.

Scholz, N.L., J. De Vente, J.W. Truman and K. Graubard. 2001. Neural network partitioning by NO and cGMP. Journal of Neuroscience 21:1610-1618.

Scholz, N.L., J.S. Labenia, J. De Vente, K. Graubard and M.F. Goy. 2002. Expression of nitric oxide synthase and nitric oxide-sensitive guanylate cyclase in the crustacean cardiac ganglion. Journal of Comparative Neurology 454:158-167.

Stasiv, Y., M. Regulski, B. Kuzin, T. Tully and G. Enikolopov. 2001. The Drosophila Nitricoxide Synthase Gene (dNOS) Encodes a Family of Proteins That Can Modulate NOS Activity by Acting as Dominant Negative Regulators. Journal of Biological Chemistry 276:42241-42251.

Torreilles, J. and M.C. Guérin. 1999. Production of peroxynitrite by zymosan stimulation of Mytilus galloprovincialis haemocytes in vitro. Fish and Shellfish Immunology 9:509518.

Truman, J.W., J. De Vente and E.E. Ball. 1996. Nitric oxide-sensitive guanylate cyclase activity is associated with the maturational phase of neuronal development in insects. Development 122:3949-3958.

Yeh, F.C., S.H. Wu, C.Y. Lai and C.Y. Lee. 2006. Demonstration of nitric oxide synthase activity in crustacean hemocytes and anti-microbial activity of hemocyte-derived nitric oxide. Comparative Biochemistry and Physiology - B Biochemistry and Molecular Biology 144:11-27. 